\title{
MEG and EEG Source Localization in Beamspace
}

\author{
Alberto Rodríguez-Rivera*, Member, IEEE, Boris V. Baryshnikov, Barry D. Van Veen, Fellow, IEEE, and \\ Ronald T. Wakai
}

\begin{abstract}
Beamspace methods are applied to EEG/MEG source localization problems in this paper. Beamspace processing involves passing the data through a linear transformation that reduces the data dimension prior to applying a desired statistical signal processing algorithm. This process generally reduces the data requirements of the subsequent algorithm. We present one approach for designing beamspace transformations that are optimized to preserve source activity located within a given region of interest and show that substantial reductions in dimension are obtained with negligible signal loss. Beamspace versions of maximum likelihood dipole fitting, MUSIC, and minimum variance beamforming source localization algorithms are presented. The performance improvement offered by the beamspace approach with limited data is demonstrated by bootstrapping somatosensory data to evaluate the variability of the source location estimates obtained with each algorithm. The quantitative benefits of beamspace processing depend on the algorithm, signal to noise ratio, and amount of data. Dramatic performance improvements are obtained in scenarios with low signal to noise ratio and a small number of independent data samples.
\end{abstract}

Index Terms-Beamspace, dipole fitting, electroencephalography, magnetoencephalography, maximum-likelihood, MUSIC, source localization, spatial filtering.

\section{INTRODUCTION}

$\mathbf{E}$ STIMATING the locations of sources of electrical activity in the brain is an important problem in electro- and magnetoencephalography (EEG and MEG). A large variety of algorithms have been developed for solving the EEG/MEG source localization problem, including multiple signal classification (MUSIC) [1]-[3], minimum variance spatial filtering methods [4]-[6], sLORETA [7], and dipole fitting [8]-[10]. The EEG/MEG source localization problem is particularly difficult because the signal-to-noise ratio (SNR) is typically very low, the noise is spatially colored and often temporally nonstationary, and the number of data samples containing the activity of interest is often very limited. Scenarios involving both low SNR and a small number of observations are common.

Localization methods that rely on estimating the secondorder statistics of the measured data or related quantities, such as signal/noise subspaces, can provide excellent performance

Manuscript received November 20, 2004; revised June 28, 2005. This work was supported in part by the National Institutes of Health (NIH) under Grant R01HL0631742 and Grant R01NS037740. Asterisk indicates corresponding author.

*A. Rodríguez-Rivera is with the Department of Electrical and Computer Engineering, University of Wisconsin-Madison, 1415 Engineering Drive, Madison, WI 53706 USA (e-mail: arod@ieee.org).

B. V. Baryshnikov and R. T. Wakai are with the Department of Medical Physics, University of Wisconsin, Madison WI 53706 (e-mail: boris@cs.wisc.edu; rtwakai@facstaff.wisc.edu).

B. D. Van Veen is with the Department of Electrical and Computer Engineering, University of Wisconsin-Madison, Madison, WI 53706 USA (e-mail: vanveen@engr.wisc.edu).

Digital Object Identifier 10.1109/TBME.2005.869764 given a sufficient number of observations. Examples of such algorithms include MUSIC [1], minimum variance beamforming (MVB) [4], [5], and maximum likelihood dipole fitting (MLDF) [10]. However, in limited data scenarios the estimated second order statistics or subspaces possess considerable variance and localization performance deteriorates. The data requirements for such algorithms generally increase as the number of spatial channels increase. For example, when using the sample covariance matrix to estimate the spatial covariance matrix of the data for MVB, the number of statistically independent data records should be three or more times the number of channels in order to obtain statistically stable source location estimates [4], [11], [12]. Consequently, the potential advantages of having large numbers of spatial channels are offset by the requirements for increased data.

Analogous problems are well known in radar and sonar statistical signal processing applications. The availability of large numbers of sensors, sometimes in the thousands, offers the potential for improved resolution of closely spaced sources and improved detection of weak sources in noise. However, these advantages are only realized with proportionate increases in data requirements. One approach for reducing the data record requirements in adaptive filtering applications is partial adaptivity, e.g., [13] and [14], in which the number of adaptive degrees of freedom is intentionally limited. A closely related approach is beamspace processing, e.g., [12] and [15]-[19], in which the sensor space data is mapped into a lower dimensional space using a linear transformation before applying the desired statistical signal processing algorithm. That is, each data vector is multiplied by a matrix that reduces the dimension of the data vector before processing. Dimension reduction is obtained by designing the beamspace transformation to focus on a limited angular sector of the range of possible source directions. The term beamspace was coined because the spatial response of the columns of the dimension-reducing matrix were often designed to form a set of overlapping directional beams, i.e., they had large gain to a set of directions spanning a desired angular sector and low gain elsewhere. The data requirements for statistical signal processing algorithms applied to the beamspace data are reduced in proportion to the reduction in dimension and the performance attributes of the original large dimension problem are not compromised for signals that originate in the angular sector spanned by the beamspace transformation.

In this paper we apply beamspace methodology to EEG/MEG source localization algorithms. The primary goal is to reduce the data sample requirements for a given performance level, although other benefits such as reduced sensitivity to correlation between sources may also result [20]. We present an approach for designing beamspace transformations that are optimized to preserve sources within a specified region of interest 
(ROI) within the head, and show that substantial reductions in dimension may be obtained with negligible performance loss for fairly large regions of the brain. Next, we illustrate application of the beamspace approach using three different source localization algorithms: MLDF, MUSIC, and MVB. We demonstrate that our design procedure introduces negligible performance loss in the asymptotic case of known statistics. The performance improvement offered by the beamspace approach with limited data is demonstrated by applying the bootstrap [21], [22] to MEG subject data obtained using somatosensory stimulation. Bootstrapping is employed to assess the variability of the source location estimates for different numbers of data records using the original and beamspace algorithms. Remarkable reductions in variability are observed in many cases, especially when the number of data records is small.

Gross and Ioannides [23] present several criteria for designing linear transformations of MEG data, one of which is similar to the method we describe here. However, the scope of this paper differs in that our purpose is not limited to transformation design, but also to assess the potential benefits of using dimension reducing transformations in a source localization context. In principle any data independent transformation may be used for beamspace processing, e.g., the signal space separation basis of Taulu, et al. [24]. Reductions in data requirements are primarily dependent on the beamspace dimension. However, any potential performance loss associated with reducing dimension, for example, loss of resolution, depends on the specific transformation design and the subsequent algorithm.

The organization of this paper is as follows. Design of beamspace transformations for a specified ROI and beamspace versions of MVB, MUSIC, and MLDF algorithms are given in Section II. The bootstrap results for determining estimated source location variability are given in Section III. Section IV contains thorough discussion of the results and Section V summarizes the paper. Bold face lower and upper case symbols denote vectors and matrices, repectively, while $\operatorname{tr}(\mathbf{A})$ and $|\mathbf{A}|$ represents the trace and determinant of $\mathbf{A}$, respectively.

\section{METHODS}

Let the $N \times 1$ vector $\mathbf{x}(j, k)$ represent the magnetic field strength measured across all $N$ sensors at epoch $j=1,2, \ldots, J$ and time $k=1,2, \ldots, K$. Assume the data consists of $L$ dipolar sources plus noise, in which case we may write

$$
\mathbf{x}(j, k)=\sum_{i=1}^{L} \mathbf{H}\left(\boldsymbol{\theta}_{i}\right) \mathbf{m}_{i}(j, k)+\mathbf{n}(j, k) .
$$

Here, $\mathbf{H}\left(\boldsymbol{\theta}_{i}\right)$ is the $N \times 3$ lead field matrix whose columns are the forward fields for unit amplitude dipoles in the $x, y$, and $z$ directions, and $\mathbf{m}_{i}(j, k)$ is the $3 \times 1$ dipole moment at location $\boldsymbol{\theta}_{i}$, epoch $j$, and time $k$. The noise is represented by $\mathbf{n}(j, k)$. Assuming the dipole moment orientation is fixed, we may write $\mathbf{m}_{i}(j, k)=$ $\mathbf{m}\left(\boldsymbol{\theta}_{i}\right) s_{i}(j, k)$, where $\mathbf{m}\left(\boldsymbol{\theta}_{i}\right)$ is the unit-norm moment orientation vector for location $\boldsymbol{\theta}_{i}$ and the scalar $s_{i}(j, k)$ describes the variation of the moment amplitude across epochs and time.

We denote the beamspace transformation using the $N \times M$ matrix $\mathbf{T}$ and obtain the $M<N$ dimensional beam space data vectors as $\mathbf{z}(j, k)=\mathbf{T}^{t} \mathbf{x}(j, k)$. We assume the columns of $\mathbf{T}$ are orthonormal without loss of generality.

\section{A. Beamspace Transformation Design}

The process of transforming into beamspace discards information in the data and, thus, can result in undesired performance characteristics, such as loss of resolution or reduced ability to detect sources, if the transformation is not chosen appropriately. In this subsection we present one method for designing $\mathbf{T}$ that is based on minimizing the average error between the original and beamspace representation of sources.

The beamspace data may be interpreted geometrically as the coordinates of the data in the space spanned by the columns of $\mathbf{T}$. Hence, the component that is not represented in beamspace is the projection of the data onto the space orthogonal to $\mathbf{T}$, i.e., $\left(\mathbf{I}-\mathbf{T T}^{t}\right) \mathbf{x}(j, k)$. This implies that the squared error associated with mapping a source at $\boldsymbol{\theta}$ into beamspace is proportional to

$$
e_{\mathbf{T}}^{2}(\boldsymbol{\theta})=\left\|\left(\mathbf{I}-\mathbf{T} \mathbf{T}^{t}\right) \mathbf{H}(\boldsymbol{\theta}) \mathbf{m}(\boldsymbol{\theta})\right\|_{2}^{2} .
$$

If the moment orientation $\mathbf{m}(\boldsymbol{\theta})$ is unknown, then we may replace $\mathbf{m}(\boldsymbol{\theta})$ in (2) with the identity matrix. In general, a good $\mathbf{T}$ will lead to a small error for all $\boldsymbol{\theta}$ in a region of interest (ROI), say $\left\{\boldsymbol{\theta} \in \Omega_{0}\right\}$. This implies that the columns of $\mathbf{T}$ approximately span the space defined by the set of forward solutions $\left\{\mathbf{H}(\boldsymbol{\theta}) \mathbf{m}(\boldsymbol{\theta}): \boldsymbol{\theta} \in \Omega_{o}\right\}$. This reasoning suggests that one criterion for choosing $\mathbf{T}$ for use with an arbitrary localization algorithm is to minimize the mean squared representation error (MSRE) over $\Omega_{0}$

$$
\min _{\mathbf{T}}\left\{\int_{\Omega_{o}} e_{\mathbf{T}}^{2}(\boldsymbol{\theta}) d \boldsymbol{\theta}\right\} .
$$

Note that if $\mathbf{T}$ is customized for a specific subsequent signal processing algorithm, then components of sources that are not exploited by the signal processing algorithm do not need to be represented by $\mathbf{T}$. Expanding the integrand using (2), we find that the minimization problem described by (3) is equivalent to the maximization problem

$$
\max _{\mathbf{T}} \operatorname{tr}\left(\mathbf{T}^{t} \mathbf{G T}\right) \text { subject to } \mathbf{T}^{t} \mathbf{T}=\mathbf{I}
$$

where

$$
\mathbf{G}=\int_{\Omega_{o}} \mathbf{H}(\boldsymbol{\theta}) \mathbf{M}(\boldsymbol{\theta}) \mathbf{H}^{t}(\boldsymbol{\theta}) d \boldsymbol{\theta}
$$

Here, $\mathbf{M}(\boldsymbol{\theta})=\mathbf{m}(\boldsymbol{\theta}) \mathbf{m}^{t}(\boldsymbol{\theta})$ for the known moment case and $\mathbf{M}(\boldsymbol{\theta})=\mathbf{I}$ if the moments are assumed unknown. In practice $\mathbf{G}$ is computed by approximating the integral with a sum. The solution to (4) is obtained by choosing the columns of $\mathbf{T}$ as the $M$ eigenvectors corresponding to the $M$ largest eigenvalues of $\mathbf{G}$. The corresponding minimum MSRE is given by the sum of the $N-M$ smallest eigenvalues of $\mathbf{G}$. A beamspace transformation designed this way effectively spans the same space as the collection of all lead field matrices in the ROI. Consequently, this beamspace transformation is applicable to any source configuration within the ROI, including extended sources. 
We normalize the MSRE by the mean energy of sources averaged over $\Omega_{o}$ to obtain an ROI independent metric for evaluating the representation quality of this beamspace transformation

$$
\tilde{e}_{\mathbf{T}}^{2}=\frac{\sum_{i=M+1}^{N} \lambda_{i}}{\operatorname{tr}(\mathbf{G})}=\frac{\left\{\sum_{i=M+1}^{N} \lambda_{i}\right\}}{\left\{\sum_{i=1}^{N} \lambda_{i}\right\}}
$$

where $\lambda_{i}, i=1,2, \ldots, N$ are the eigenvalues of $\mathbf{G}$ arranged in descending order. This metric can be interpreted as the fraction of source energy not represented by the beamspace transformation. Values of $\tilde{e}_{T}^{2}$ that are very close to zero indicate that the beamspace transformation accurately represents sources in the ROI. This occurs if $\mathbf{G}$ corresponds to an approximately low rank matrix. Note that $\tilde{e}_{\mathbf{T}}^{2}$ is a "global" metric of performance since it represents average error over the entire ROI.

A local measure of the beamspace transformation's ability to represent sources is given by the ratio of the beamspace to the original source energies at each location

$$
F_{\mathbf{T}}(\boldsymbol{\theta})=\frac{\operatorname{tr}\left(\mathbf{T}^{t} \mathbf{H}^{t}(\boldsymbol{\theta}) \mathbf{M}(\boldsymbol{\theta}) \mathbf{H}(\boldsymbol{\theta}) \mathbf{T}\right)}{\operatorname{tr}\left(\mathbf{H}^{t}(\boldsymbol{\theta}) \mathbf{M}(\boldsymbol{\theta}) \mathbf{H}(\boldsymbol{\theta})\right)} .
$$

Note that each column of the beamspace transformation may be viewed as a spatial filter. $F_{\mathbf{T}}(\boldsymbol{\theta})$ describes the normalized sum of the gains of the individual columns of $\mathbf{T}$ to the source and, thus, represents the effective spatial filtering function of the beamspace transformation. An ideal $F_{\mathbf{T}}(\boldsymbol{\theta})$ is exactly one on the ROI (passband) and exactly zero elsewhere (stopband). While a value near unity in the ROI is obtained by choosing $M$ such that $\tilde{e}_{\mathbf{T}}^{2}$ is near zero, in general the response outside the ROI is nonzero, as the examples in Section III-A-1 illustrate.

There are many different criteria that could be chosen to design the beamspace transformation, some of which may be customized to the specific application. For example, if correlated sources are present in a known region outside the ROI, then the beamspace transformation could be chosen to minimize the average gain in the correlated source region subject to a constraint on the average MSRE in the ROI [see (3)] to suppress the effect of the correlated sources on localization in the ROI. Dalal, et al. [25] recently demonstrated a closely related idea of using null constraints with MVB to suppress correlated activity in one hemisphere for auditory evoked response data. Similar ideas can be used to design beamspace transformations that attenuate external interference such as heart beats. Toward this end, Talu, $e t$ al. [24] obtain a basis for signals that originate from within a sphere enclosing the brain. A variety of additional criteria that could be applied to beamspace transformation design are given in [23]. Note that in order to preserve the reduction in statistical variability associated with mapping into beamspace, $\mathbf{T}$ should be chosen in a data-independent fashion. If $\mathbf{T}$ is designed from the data, then the randomness in $\mathbf{T}$ will introduce an additional degree of variability to the subsequent localization algorithm that may negate the potential benefits of reducing the dimension of the source localization problem.

\section{B. Beamspace Source Localization Algorithms}

Beamspace source localization algorithms operate on the beamspace data $\mathbf{z}(j, k)$. In general they are identical to their sensor space counterparts, with the exception that the lead field matrix must be mapped into beamspace to obtain
$\mathbf{H}_{\mathbf{T}}(\boldsymbol{\theta})=\mathbf{T}^{t} \mathbf{H}(\boldsymbol{\theta})$. Let the columns of $\mathbf{U}_{\mathbf{T}}(\boldsymbol{\theta})$ be an orthonormal basis for the space spanned by the columns of $\mathbf{H}_{\mathbf{T}}(\boldsymbol{\theta})$. The rank of $\mathbf{H}_{\mathbf{T}}(\boldsymbol{\theta})$ is three in general, although if a spherical head model is used in an MEG application the rank is two. A source is modeled as $\mathbf{U}_{\mathbf{T}}\left(\boldsymbol{\theta}_{i}\right) \tilde{\mathbf{m}}\left(\boldsymbol{\theta}_{i}\right) s_{i}(j, k)$ where $\tilde{\mathbf{m}}(\boldsymbol{\theta})$ is a unit-norm vector that describes the unknown moment orientation with respect to the basis $\mathbf{U}_{\mathbf{T}}(\boldsymbol{\theta})$ for the beamspace lead field matrix. The algorithms presented in the following subsections assume the moment orientation $\mathbf{m}(\boldsymbol{\theta})$ or $\tilde{\mathbf{m}}(\boldsymbol{\theta})$ is fixed but unknown. We only present beamspace versions of the algorithms for a dipolar model. It is straightforward to extend the algorithms to other parametric models for the spatial component of the signal, including rotating dipoles. Sensor space versions of the algorithms are obtained using $\mathbf{T}=\mathbf{I}$.

Define the $M \times K$ beamspace-time data matrix for the $j$ th epoch as

$$
\mathbf{Z}_{j}=\left[\begin{array}{llll}
\mathbf{z}(j, 0) & \mathbf{z}(j, 1) & \ldots & \mathbf{z}(j, K-1)
\end{array}\right] .
$$

We concatenate the data from all epochs into an $M$ by $J K$ matrix

$$
\mathbf{Z}=\left[\begin{array}{llll}
\mathbf{Z}_{1} & \mathbf{Z}_{2} & \ldots & \mathbf{Z}_{J}
\end{array}\right] .
$$

The sample covariance matrix of the beamspace data is expressed as

$$
\begin{aligned}
\mathbf{C}(\mathbf{Z}) & =\frac{1}{J K-1} \sum_{j=1}^{J} \sum_{k=1}^{K} \mathbf{z}(j, k) \mathbf{z}^{t}(j, k) \\
& =\frac{1}{J K-1} \mathbf{Z Z}^{t} .
\end{aligned}
$$

Source localization in all three algorithms is based on finding the minimum or maximum of a data dependent cost function. The search for an extremum is performed by first evaluating the cost function on the surface of the cortex, which is determined from the subject's magnetic resonance image using the FreeSurfer package [26]. In the second stage the source location is refined using a bounded unconstrained numerical optimization initialized with the extremum found on the cortical surface.

1) MLDF: The MLDF approach of Dogandzic and Nehorai [10] exploits the temporal multiepoch structure of evoked response data to estimate the spatial noise covariance matrix from the samples of data that are being localized, thus avoiding the assumption that the noise statistics are identical prestimulus and poststimulus.

Assuming the signal to be localized is identical in each epoch, we express $\mathbf{Z}_{j}=\mathbf{S}+\mathbf{N}_{j}$ where $\mathbf{S}$ is the signal component. We further assume that we are localizing a single dipolar source and that the temporal evolution of the signal lies in a known frequency band so that $\mathbf{S}$ is expressed as

$$
\mathbf{S}=\mathbf{U}_{\mathbf{T}}(\boldsymbol{\theta}) \boldsymbol{\mu} \mathbf{C}^{t}
$$

where the $2 \times L$ (or $3 \times L$ ) matrix $\mu$ represents unknown amplitude parameters and the columns of the $K \times L$ matrix $\mathbf{C}$ are a known basis for the temporal evolution of the signal. In this paper we use knowledge of the frequency band of interest to construct $\mathbf{C}$ following the procedure presented in [27]. Dogandzic and Nehorai [10] consider two different models for the temporal basis-one in which the temporal basis is completely unstructured and estimated from the data, and a second in which 
the temporal basis is a function of several unknown parameters that are estimated in the maximum likelihood procedure. Our approach of using the frequency band of interest to determine a fixed $\mathbf{C}$ is shown to be very effective with real data in the related problem of estimating $\mathbf{S}$ [27].

Since the signal component is repeated in each epoch, we write

$$
\mathbf{Z}=\left[\begin{array}{llll}
\mathbf{S} & \mathbf{S} & \ldots & \mathbf{S}
\end{array}\right]+\left[\begin{array}{llll}
\mathbf{N}_{1} & \mathbf{N}_{2} & \ldots & \mathbf{N}_{J}
\end{array}\right] .
$$

The signal component is rewritten using (12) as $\left[\begin{array}{llll}\mathbf{S} & \mathbf{S} & \ldots & \mathbf{S}\end{array}\right]=\mathbf{U}_{\mathbf{T}}(\boldsymbol{\theta}) \boldsymbol{\mu} \mathbf{D}^{t}$ where the multiepoch temporal basis matrix $\mathbf{D}^{t}=\left[\begin{array}{llll}\mathbf{C}^{t} & \mathbf{C}^{t} & \ldots & \mathbf{C}^{t}\end{array}\right]$. We assume the columns of the noise matrices $\mathbf{N}_{j}, j=1,2, \ldots J$ are independent, identically distributed zero-mean Gaussian random vectors with unknown covariance matrix. This implies that noise is spatially colored and approximately white temporally. The signal amplitude matrix $\boldsymbol{\mu}$ and noise covariance matrix are nuisance parameters that must be estimated in order to obtain the maximum likelihood estimate of the source location $\boldsymbol{\theta}$.

Substituting the maximum likelihood estimates of the noise spatial covariance matrix and signal amplitude parameters into the likelihood function (see [10], [28]) results in the following problem for obtaining the maximum likelihood estimate of $\boldsymbol{\theta}$

$$
\begin{aligned}
\min _{\boldsymbol{\theta}} \mid \mathbf{I}+\mathbf{Z}_{\mathbf{D}}^{t} \mathbf{Q}_{\overline{\mathbf{D}}}^{-1} \mathbf{Z}_{\mathbf{D}}-\mathbf{Z}_{\mathbf{D}}^{t} \mathbf{Q}_{\overline{\mathbf{D}}}^{-1} \mathbf{U}_{\mathbf{T}}(\boldsymbol{\theta}) \\
\times\left(\mathbf{U}_{\mathbf{T}}^{t}(\boldsymbol{\theta}) \mathbf{Q}_{\overline{\mathbf{D}}}^{-1} \mathbf{U}_{\mathbf{T}}(\boldsymbol{\theta})\right)^{-1} \mathbf{U}_{\mathbf{T}}^{t}(\boldsymbol{\theta}) \mathbf{Q}_{\overline{\mathbf{D}}}^{-1} \mathbf{Z}_{\mathbf{D}} \mid
\end{aligned}
$$

where we have defined the following quantities:

$$
\mathbf{Z}_{\mathbf{D}}=\mathbf{Z D}\left(\mathbf{D}^{t} \mathbf{D}\right)^{-\frac{1}{2}}
$$

and

$$
\mathbf{Q}_{\bar{D}}=\mathbf{Z}_{\overline{\mathbf{D}}} \mathbf{Z}_{\overline{\mathbf{D}}}^{t}
$$

Here, $\mathbf{Z}_{\bar{D}}=\mathbf{Z} \overline{\mathbf{D}}$ where the columns of the $J K \times(J K-L)$ matrix $\mathbf{D}$ are an orthonormal basis for the space orthogonal to that spanned by the columns of $\mathbf{D}$. Thus, $\mathbf{Z}_{\overline{\mathbf{D}}}$ contains only noise and $\mathbf{Q}_{\overline{\mathbf{D}}}$ is proportional to the spatial sample covariance matrix of the noise (see, e.g, [27]). Localization is achieved by finding the $\theta$ that minimizes the cost function in (14).

2) MUSIC: The MUSIC algorithm uses the data to estimate a signal plus noise subspace and the noise only subspace. The fact that forward solutions corresponding to sources in the data are orthogonal to the noise only subspace is exploited to obtain a cost function whose peaks represent estimated source locations. Identification of the noise only subspace is performed using the singular value decomposition of the beamspace data matrix $\mathbf{Z}$ or equivalently from the eigendecomposition of the beamspace sample covariance matrix $\mathbf{C}(\mathbf{Z})$. If the noise is spatially white, then the noise only space is spanned by the left singular/eigen vectors associated with the smallest singular/eigen values. We collect these singular/eigen vectors into a unitary matrix $\boldsymbol{\Phi}_{n}$. Methods for determining the number of noise only singular/eigen vectors are discussed in [1]. In practice the noise is often colored and MUSIC may be preceded by a whitening step based on an estimated noise covariance matrix [3]. We use $\mathbf{Q}_{\overline{\mathrm{D}}}$ defined in (16) to spatially whiten the data before calculating the MUSIC function.
We choose the unknown moment orientation $\tilde{\mathbf{m}}(\boldsymbol{\theta})$ to maximize the orthogonality between the noise subspace $\boldsymbol{\Phi}_{n}$ and the signal component $\tilde{\mathbf{U}}_{\mathbf{T}}(\boldsymbol{\theta}) \tilde{\mathbf{m}}(\boldsymbol{\theta})$ where $\tilde{\mathbf{U}}_{\mathbf{T}}(\boldsymbol{\theta})$ is an orthonormal basis for the whitened, beamspace leadfield matrix $\mathbf{Q}_{\overline{\mathbf{D}}}^{-1 / 2} \mathbf{T}^{t} \mathbf{H}(\boldsymbol{\theta})$. This results in the MUSIC cost function

$$
\operatorname{MUSIC}_{\mathbf{T}}(\boldsymbol{\theta})=\frac{1}{\lambda_{\min }^{\mathrm{MUSIC}}(\boldsymbol{\theta})}
$$

where $\lambda_{\min }^{\mathrm{MUSIC}}(\boldsymbol{\theta})$ is the minimum eigenvalue of the matrix $\tilde{\mathbf{U}}_{\mathbf{T}}^{t}(\boldsymbol{\theta}) \boldsymbol{\Phi}_{n} \boldsymbol{\Phi}_{n}^{t} \tilde{\mathbf{U}}_{\mathbf{T}}(\boldsymbol{\theta})$. In this paper we consider localizing a single source, so we choose the source location as the $\theta$ that maximizes $\operatorname{MUSIC}_{\mathbf{T}}(\boldsymbol{\theta})$.

3) MVB: MVB localizes the source based on the output power of a spatial filter constrained to pass activity from a location of interest while minimizing the variance at the filter output. The location of interest is scanned to produce a metric of source activity as a function of location. Let $\mathbf{w}_{\mathbf{T}}(\boldsymbol{\theta})$ denote the beamspace spatial filter weights for location $\theta$. The MVB spatial filter is obtained by solving

$\min _{\mathbf{w}_{\mathbf{T}}(\boldsymbol{\theta})} E\left\{\left|\mathbf{w}_{\mathbf{T}}^{t}(\boldsymbol{\theta}) \mathbf{z}(j, k)\right|^{2}\right\}$ subject to $\mathbf{w}_{\mathbf{T}}^{t}(\boldsymbol{\theta}) \mathbf{U}_{\mathbf{T}}(\boldsymbol{\theta}) \tilde{\mathbf{m}}(\boldsymbol{\theta})=1$

where $\tilde{\mathbf{m}}(\boldsymbol{\theta})$ is unknown. The variance or power at the beamspace spatial filter output is expressed as a function of $\tilde{\mathbf{m}}(\boldsymbol{\theta})$ as

$$
P_{\mathbf{T}}(\boldsymbol{\theta})=\frac{1}{\tilde{\mathbf{m}}^{t}(\boldsymbol{\theta}) \mathbf{U}_{\mathbf{T}}^{t}(\boldsymbol{\theta}) \mathbf{R}_{\mathbf{z}}^{-1} \mathbf{U}_{\mathbf{T}}(\boldsymbol{\theta}) \tilde{\mathbf{m}}(\boldsymbol{\theta})}
$$

where $\mathbf{R}_{\mathbf{z}}=E\left\{\mathbf{z}(j, k) \mathbf{z}^{t}(j, k)\right\}$ is the beamspace data covariance matrix. In practice $\mathbf{R}_{\mathbf{z}}$ is unknown, so we approximate $\mathbf{R}_{\mathbf{z}}$ using the sample covariance matrix $\mathbf{C}(\mathbf{Z})$. The unknown moment orientation is chosen to maximize $P_{\mathbf{T}}(\boldsymbol{\theta})$ subject to the constraint $\tilde{\mathbf{m}}^{t}(\boldsymbol{\theta}) \tilde{\mathbf{m}}(\boldsymbol{\theta})=1$, which results in

$$
P_{\mathbf{T}}(\boldsymbol{\theta})=\frac{1}{\lambda_{\min }^{\mathrm{MVB}}(\boldsymbol{\theta})}
$$

where $\lambda_{\min }^{\mathrm{MVB}}(\boldsymbol{\theta})$ is the smallest eigenvalue of the matrix $\mathbf{U}_{\mathbf{T}}^{t}(\boldsymbol{\theta}) \mathbf{C}^{-1}(\mathbf{Z}) \mathbf{U}_{\mathbf{T}}(\boldsymbol{\theta})$.

Localization is usually performed by finding the maxima of a normalized version of the spatial filter output power. Several different normalization methods have been proposed [4], [6], [29]. In this paper, we normalize $P_{\mathbf{T}}(\boldsymbol{\theta})$ by the estimated output power due to the noise to obtain the localization cost function

$$
\operatorname{MVB}_{\mathbf{T}}(\boldsymbol{\theta})=\frac{\lambda_{\min }^{\mathrm{noise}}(\boldsymbol{\theta})}{\lambda_{\min }^{\operatorname{MVB}}(\boldsymbol{\theta})}
$$

where $\lambda_{\min }^{\text {noise }}(\boldsymbol{\theta})$ is the smallest eigenvalue of the matrix $\mathbf{U}_{\mathbf{T}}^{t}(\boldsymbol{\theta}) \mathbf{Q}_{\overline{\mathbf{D}}}^{-1} \mathbf{U}_{\mathbf{T}}(\boldsymbol{\theta})$ and $\mathbf{Q}_{\bar{D}}$ is defined in (16). Note that $1 / \lambda_{\min }^{\text {noise }}(\boldsymbol{\theta})$ is proportional to the spatial filter output power if the data consists of only noise. We choose this particular metric because it appeares to give the best overall results with the data set used in Section III. Localization of a single source is performed by finding the $\boldsymbol{\theta}$ that maximizes $\operatorname{MVB}_{\mathbf{T}}(\boldsymbol{\theta})$.

\section{RESULTS}

The cortical surface of the subject is extracted from a magnetic resonance image of the subject's brain using the FreeSurfer software [26]. The pial surface is used for both 


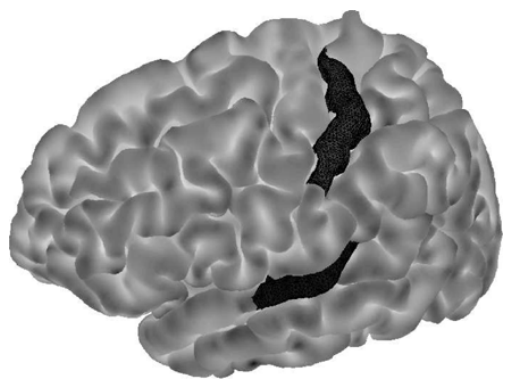

Fig. 1. Pial surface of the subject's left hemisphere. The shaded regions correspond to the ROIs identified in the somatosensory (upper) and auditory (lower) cortices.

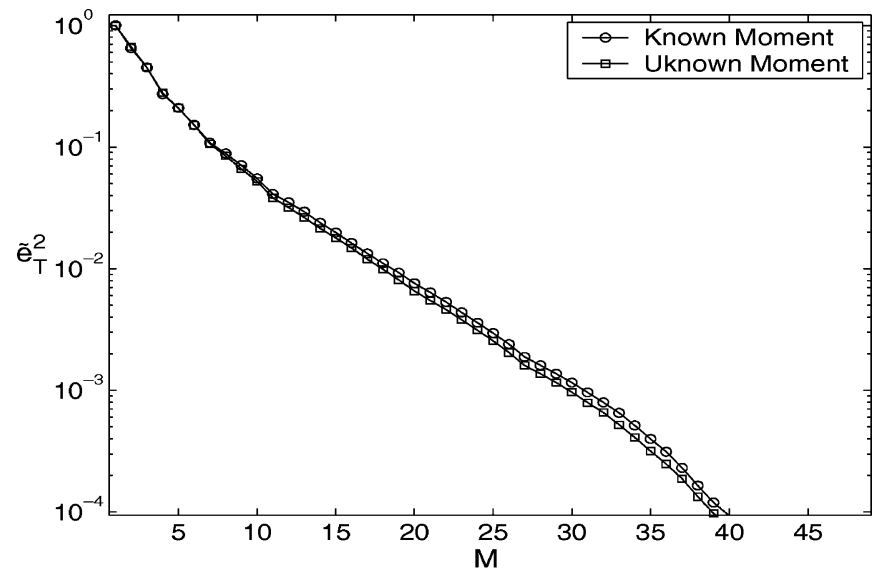

(a)

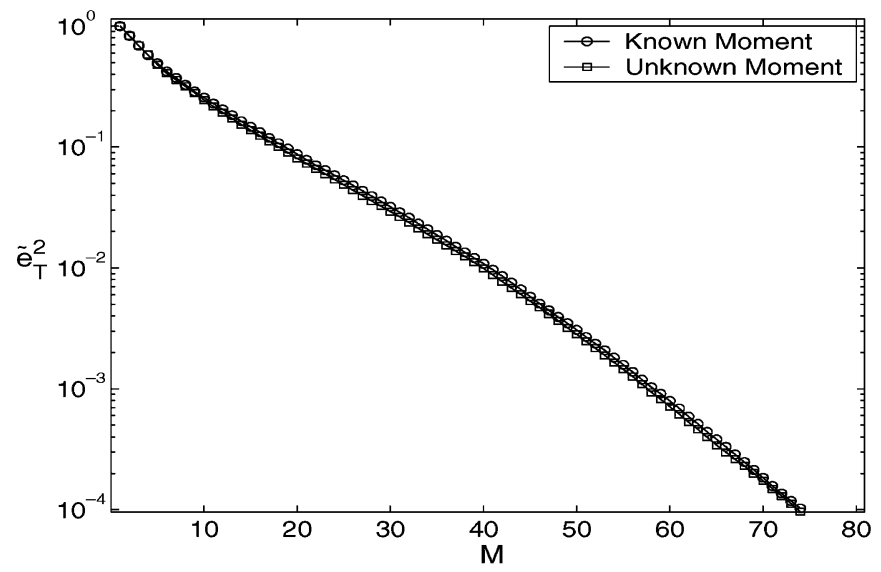

(b)

Fig. 2. Normalized MSRE (see (6)) as a function of beamspace dimension $M$ assuming the ROI is the entire left hemisphere. Both known and unknown dipole moment designs are depicted. (a) 74-channel Magnes II. (b) 248-channel Magnes 3600.

defining the ROI employed to design the beamspace transformation and to constrain the initial scanning phase of the source localization algorithms. Two different MEG systems are employed to illustrate beam space design, the 74 channel Magnes II Biomagnetometer in the University of Wisconsin Biomagnetism Laboratory and a 248 channel Magnes 3600 Whole Head system. The subject data used to evaluate beamspace localization performance is collected using the 74 channel Magnes II Biomagnetometer in a magnetically shielded room. The 74-channel system is composed of two separate 37-channel dewars. The lead field matrices are calculated for the 74-channel

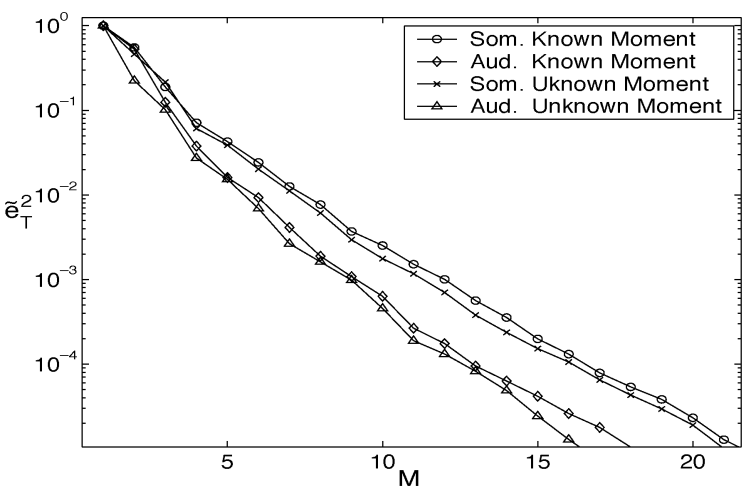

(a)

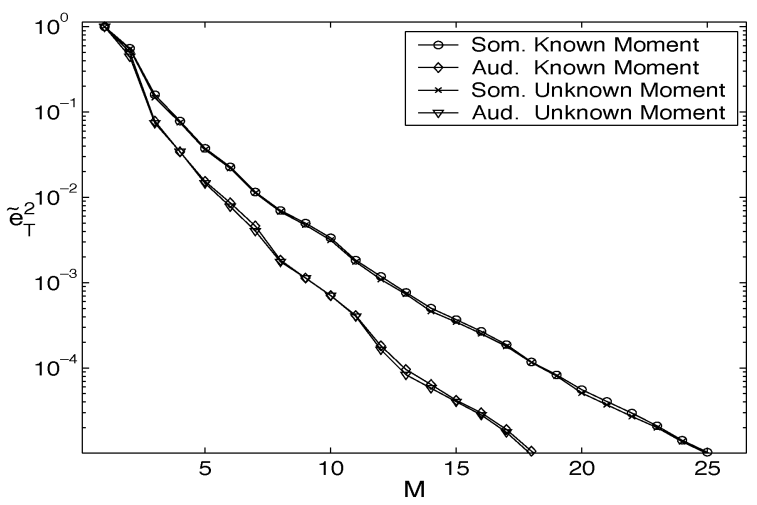

(b)

Fig. 3. Normalized MSRE (see (6)) as a function of beamspace dimension $M$ assuming the ROI is in either the somatosensory or auditory cortex for known and unknown dipole moment designs. (a) 74-channel Magnes II. (b) 248-channel Magnes 3600.

system using a dual symmetrical spherical shell model, with one sphere for each 37-channel sensor set [30]. The best fit for the sphere locations is determined based on head shape information collected during data acquisition. A single sphere model is used for the 248-channel system.

\section{A. Beamspace Transformation Design}

We consider several different ROIs to illustrate the degree of dimension reduction that can be obtained with the design procedure presented in Section II-A. Fig. 1 depicts the left hemisphere of the subject with the somatosensory and auditory ROIs shaded. The normalized MSRE defined in (6) is depicted as a function of beamspace dimension $M$ in Fig. 2 assuming the ROI is the entire left hemisphere. Note that knowledge of the moment orientation does not significantly change the normalized MSRE. A normalized MSRE of $0.1 \%\left(\tilde{e}_{\mathbf{T}}^{2}=0.001\right)$ is obtained when $M=30$ and $M=58$ for the 74- and 248-channel configurations, respectively. Fig. 3 depicts the normalized MSRE as a function of beamspace dimension when the ROI is in the auditory or somatosensory cortex. In this case a normalized MSRE of $0.1 \%$ is obtained for the somatosensory ROI when $M=13$ for both the 74- and 248-channel configurations. The auditory ROI requires $M=10$ to achieve normalized MSRE of $0.1 \%$ for both 74-channel and 248-channel configurations. Again, use of the moment orientation does not have a significant impact on the normalized MSRE. Note that the required beamspace dimension for a given normalized MSRE is not directly proportional to the number of spatial channels in the MEG system. 


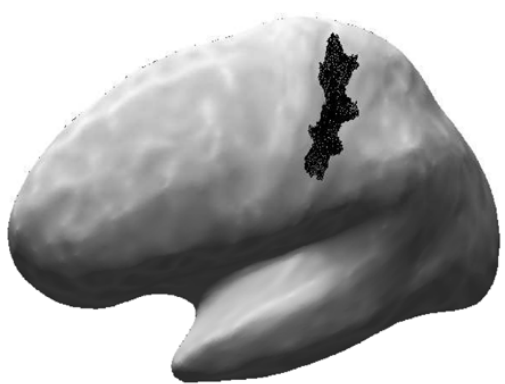

(a)

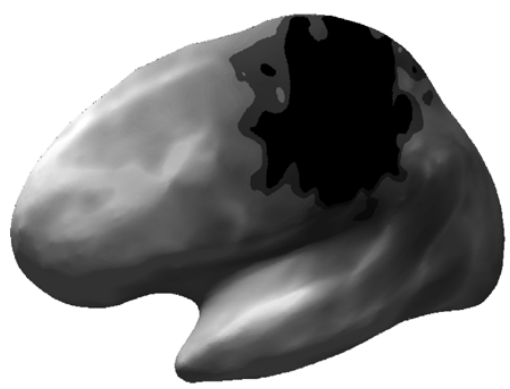

(c)

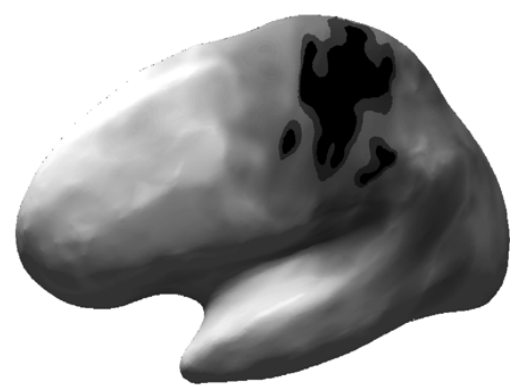

(b)

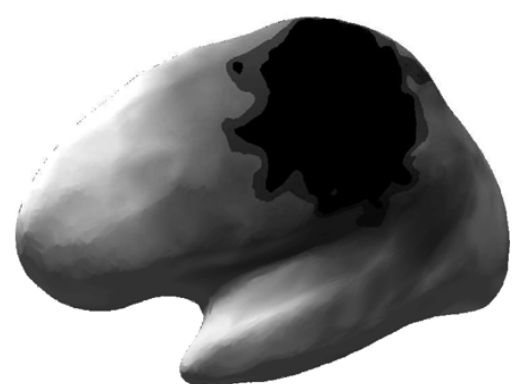

(d)

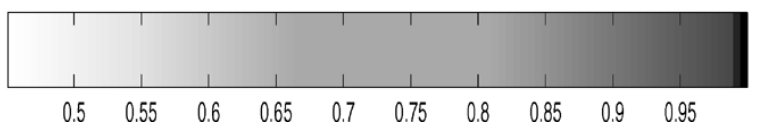

Fig. 4. Spatial filtering functions $F_{\mathbf{T}}(\boldsymbol{\theta})$ (see (7)) for the unknown moment somatosensory ROI beamspace transformations depicted on the inflated cortical surface. The scale applies to (b)-(d). (a) Representation of the somatosensory ROI on the inflated cortical surface. (b) 74 -channel configuration and $M=8$

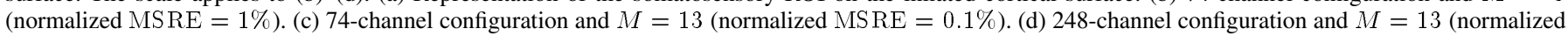
MSRE $=0.1 \%)$.

Example spatial filtering functions $F_{\mathbf{T}}(\boldsymbol{\theta})$ defined in (7) for the unknown moment somatosensory ROI beamspace transformations are depicted in Fig. 4. The spatial filtering function is near unity within the ROI, implying perfect source representation over the entire somatosensory region in all cases.

The effectiveness of the beamspace transformation design procedure is further illustrated by comparing the sensor space and beamspace MVB cost functions for a localization scenario in which the true data covariance matrix is known. Use of the known data covariance matrix represents the asymptotic case where the covariance matrix is perfectly estimated. Hence, any difference between the sensor space and beamspace cost functions is not associated with covariance matrix estimation effects, but instead is solely a consequence of the beamspace transformation discarding information. Two dipolar sources are placed in the somatosensory region and spatially white noise is added to the data. The MVB cost function (21) is evaluated on the somatosensory region for the 74-channel Magnes II configuration. Fig. 5 depicts the 74-channel sensor space cost function, a cost function based on the $M=13$ beamspace transformation with response depicted in Fig. 4(c), and two ad hoc "beamspace" cost functions - one where only the 37 channels positioned over the left hemisphere are used, and a second where the 13 channels with the strongest measured signal power are used. There is no visual difference between MVB cost functions for the 74-channel sensor space and the $M=13$ beamspace transformation designed as described in Section II-A. However, the cost functions for the two-dimension reduction methods based on throwing away some sensor channels [Fig. 5(c), (d)] shows significant loss of resolution.
1) Beamspace Source Localization Performance: The source localization performance of the beamspace MLDF, MUSIC, and MVB algorithms is compared to their sensor space counterparts using evoked response data collected with the 74-channel Magnes II Biomagnetometer. The right index finger of a healthy adult female is stimulated with a pneumatic stimulus generator, which applies a pressure pulse. Three-hundred 500-ms epochs are collected at a $520.8-\mathrm{Hz}$ sampling rate. Each epoch includes $100 \mathrm{~ms}$ prestimulus intervals. Online $0.1-\mathrm{Hz}$ high-pass and 100-Hz low-pass filters are used during data collection. The data used for MUSIC and MVB localization is filtered offline with a 4th order zero phase Butterworth filter having a 1- to 30-Hz passband. The data used for MLDF localization is not filtered offline since the temporal basis vectors in $\mathbf{C}$ effectively function as filters for the signal component of the data. Fig. 6 depicts the average of all 300 epochs of the data in butterfly format after offline filtering and identifies the portions of the data used for source localization.

The performance of all three sensor space and beamspace algorithms is evaluated as a function of the number of data samples by using 100,150, and 300 epochs of data for source localization. Furthermore, beamspace algorithms are also evaluated with only 50 epochs. All of the beamspace algorithms employ the $M=13$ transformation designed for the somatosensory ROI as described in the previous subsection and whose response is illustrated in Fig. 4(c). Two different data selection procedures are evaluated for each scenario: using a single time sample from each epoch near the peak of the average response (single time point (ST) in Fig. 6) and using 21 time samples from each epoch in the range 67.2-105.6 ms [multiple time point 


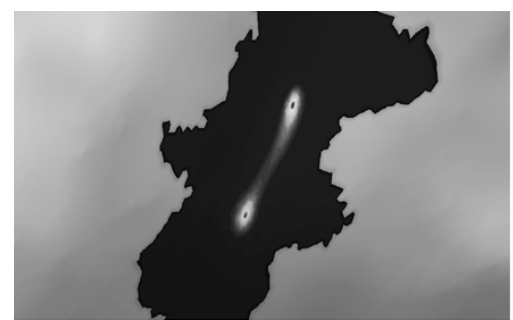

(a)

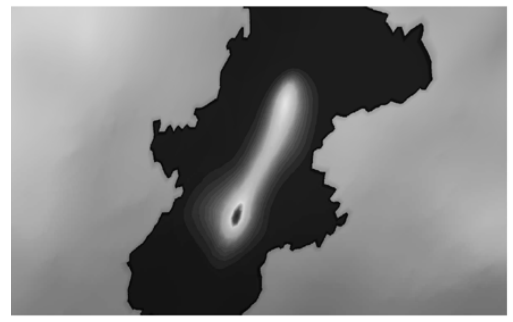

(c)

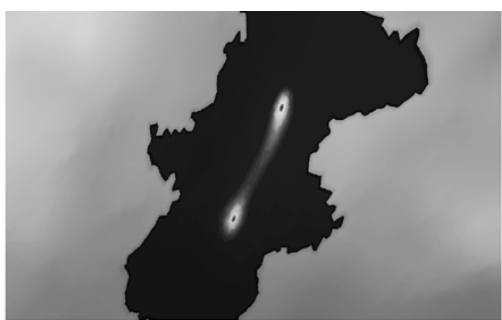

(b)

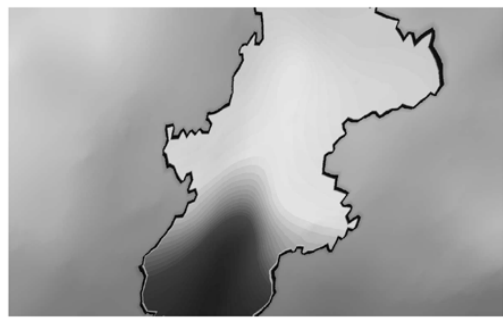

(d)

Fig. 5. MVB cost function (21) for the 74-channel Magnes II sensor system using the true covariance matrix for two dipolar sources located in the somatosensory region plus white noise. (a) 74-channel sensor space. (b) $M=13$ beamspace designed as in Section II-A. (c) $M=37$ beamspace obtained by choosing the 37 channels located over the left hemisphere. (d) $M=13$ beamspace obtained by choosing the 13 channels with the largest power.

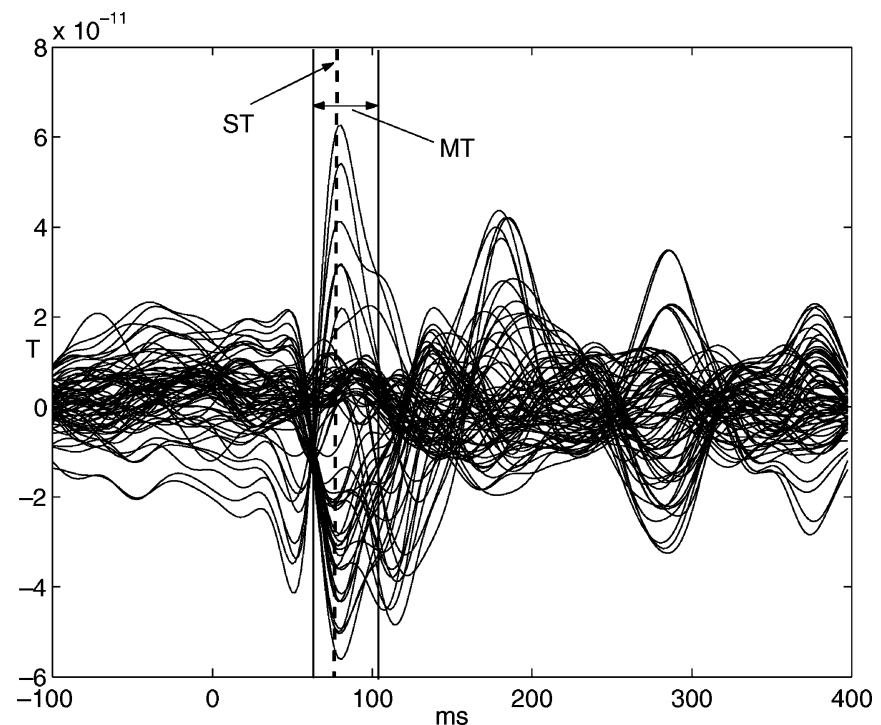

Fig. 6. Average of 300 epochs of somatosensory evoked response after bandpass filtering with a $1-$ to $30-\mathrm{Hz}$ passband. The dashed line indicates the data selected for the ST case while the solid lines indicate the time interval selected for the MT case.

(MT)] in Fig. 6). The boostrap [21], [22] method is used to assess the variability of the estimated source location for each combination of algorithms and number of epochs. Bootstrapping is a procedure for generating sample statistics from a single data set by resampling the data set with replacement. In this paper the available 300 epochs of data are resampled with replacement 1000 times for each algorithm/number of epochs combination to obtain an estimate of the mean source location and the variability of the localization algorithm about the mean.

We use $\mathbf{Q}_{\overline{\mathbf{D}}}$ defined in (16) and computed using all 300 epochs of data to account for the effects of spatially colored noise in MUSIC and MVB. This avoids the assumption that the noise statistics are the same prestimulus and poststimulus, since $\mathbf{Q}_{\overline{\mathbf{D}}}$ is calculated from the signal-free component of the data on the same time interval as the source of interest.
The noise subspace dimension in the sensor space version of MUSIC is chosen as 70 using the procedure given in [1], [2]. This corresponds to $94.6 \%$ of the available dimensions. In beamspace MUSIC the noise subspace dimension is chosen as 12 , which represents $92.3 \%$ of the available dimensions and approximately corresponds to the sensor space dimension ratio. Our experience indicates that keeping the ratio of noise subspace dimension to available dimensions approximately constant gives good results for beamspace MUSIC. If the beamspace noise subspace dimension is too small, for example, as occurs with a constant signal plus noise subspace dimension, then spurious peaks result in the MUSIC cost function.

The primary purpose of this study is to compare the variability of the source location estimates for sensor and beamspace algorithms. Fig. 7 depicts the results of performing 1000 bootstrap localizations for sensor and beamspace versions of all three algorithms using the ST case and different numbers of epochs. The scatter of the localizations shown in Fig. 7 provides a qualitative assessment of variability. Variability is quantitatively assessed by calculating the mean source location over all 1000 bootstrap resamples and then recording the distance from all 1000 estimates to the mean location. Note that the mean location is potentially different for each scenario. Figs. 8-10 present the percentages of the 1000 resamples that are localized within 1 $\mathrm{cm}$ of the mean location as a function of the number of epochs for MLDF, MUSIC, and MVB algorithms, respectively. This measures localization consistency for each estimator across the resamples. The 50 epoch sensor space results for MVB and MLDF are not computed because the estimated covariance matrix is singular. In this case the sensor space MVB and MLDF algorithms cannot be evaluated because the covariance matrix is not invertible.

Figs. 8-10 show that the consistency of the localization estimates is improved by beamspace processing in all cases. The greatest improvement occurs with small numbers of epochs, and considerably less improvement is evident when 300 epochs 


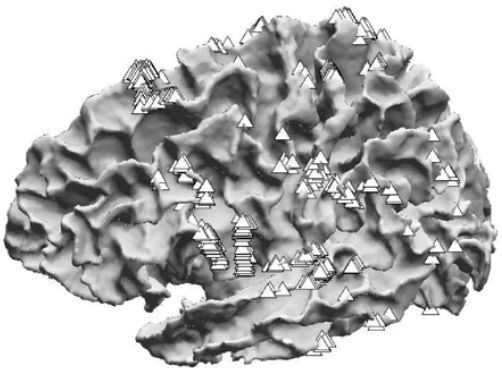

(a)

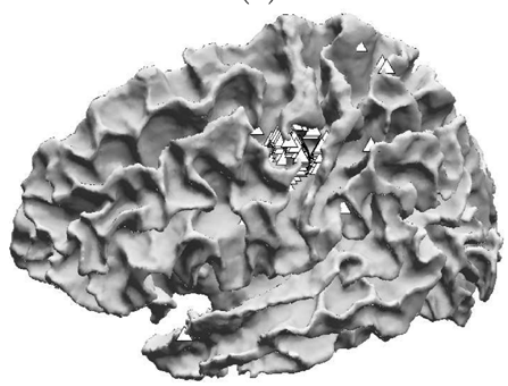

(c)

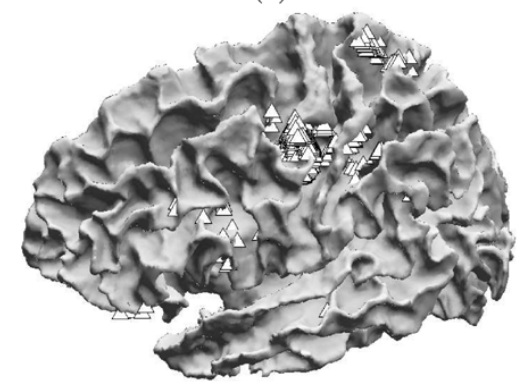

(e)

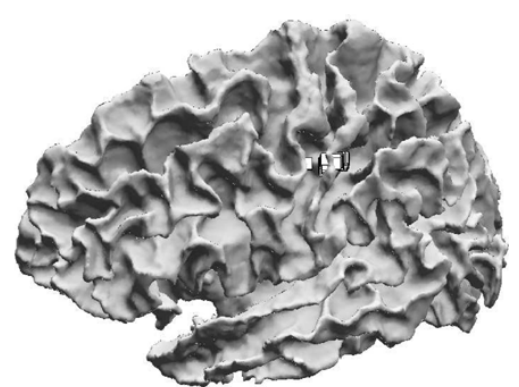

(b)

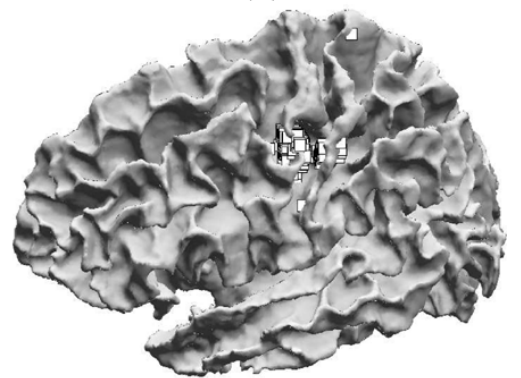

(d)

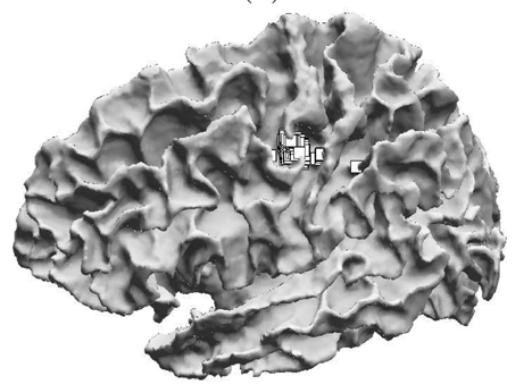

(f)

Fig. 7. Scatter plot depicting the results of 1000 bootstrap source localizations for the ST case. The sensor space results are on the left half of the figure and the corresponding beamspace results are on the right half. All beamspace examples use $M=13$. (a) Sensor space MLDF 300 epochs. (b) Beamspace MLDF 300 epochs. (c) Sensor space MUSIC 100 epochs. (d) Beamspace MUSIC 100 epochs. (e) Sensor space MVB 150 epochs. (f) Beamspace MVB 150 epochs.

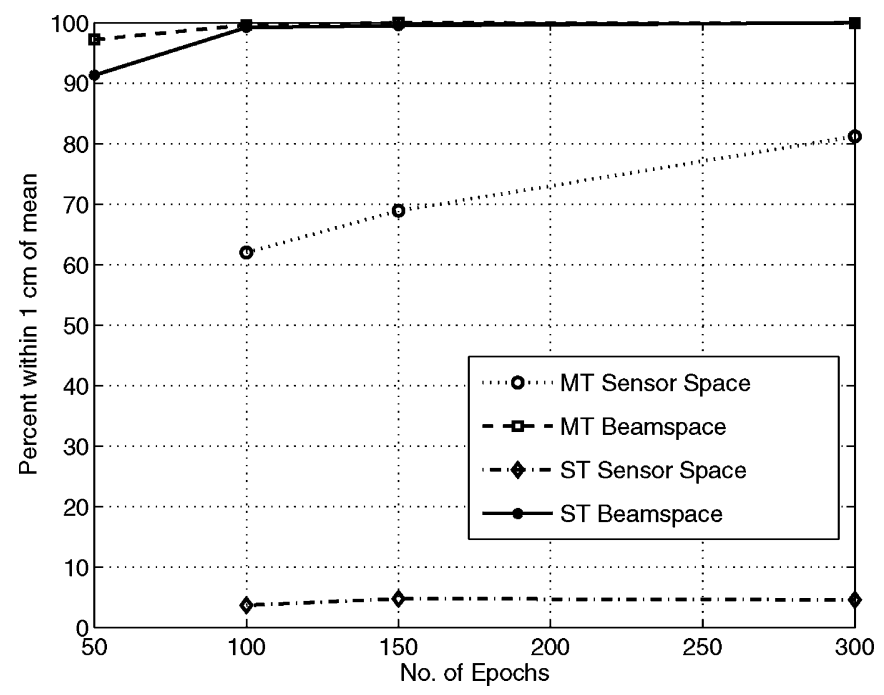

Fig. 8. Percentage of bootstrap resamples for which the source is localized within $1 \mathrm{~cm}$ of the mean location for the sensor space/beamspace MLDF methods.

are employed. The relative performance of MT versus ST and beamspace versus sensor space varies across the three algorithms and is addressed in the following section.

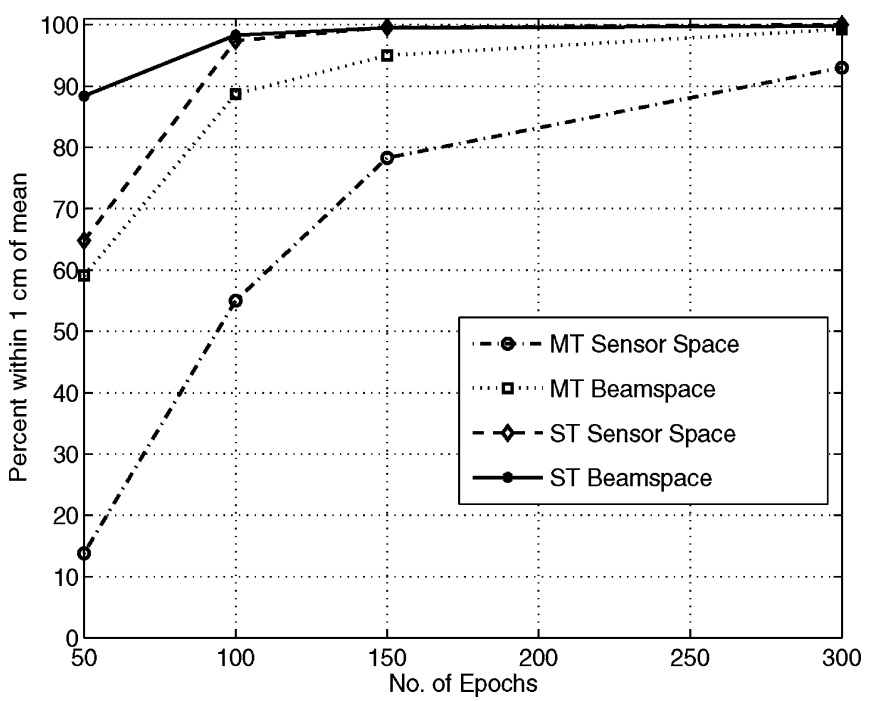

Fig. 9. Percentage of bootstrap resamples for which the source is localized within $1 \mathrm{~cm}$ of the mean location for the sensor space/beamspace MUSIC methods.

The mean source locations for all three algorithms in the ST 300 epoch case are depicted in Fig. 11. This case has the least variability averaged across all three algorithms. The mean 


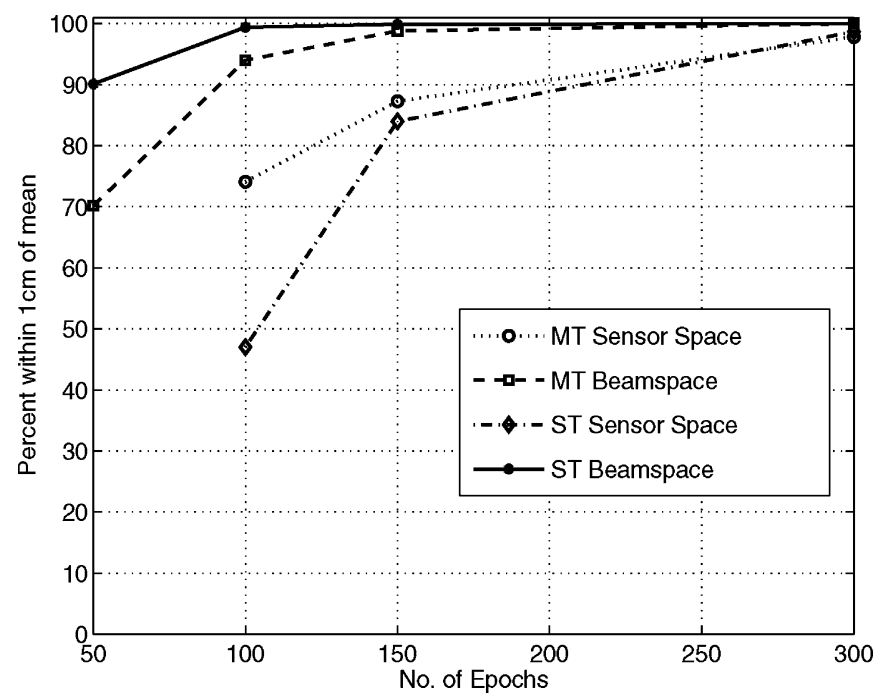

Fig. 10. Percentage of bootstrap resamples for which the source is localized within $1 \mathrm{~cm}$ of the mean location for the sensor space/beamspace MVB methods.

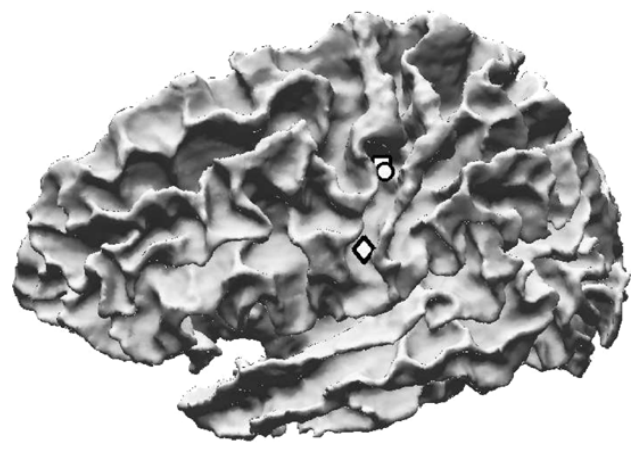

(a)

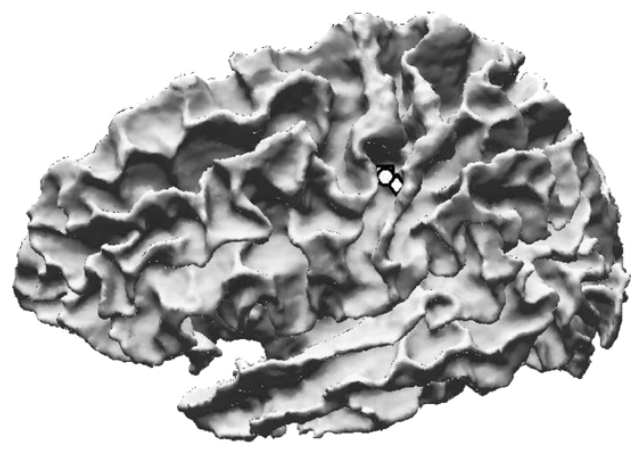

(b)

Fig. 11. Mean source locations for the ST 300 epoch case: MLDF-diamond marker, MUSIC-circle marker, MVB-square marker. a) Sensor space algorithms. b) Beamspace algorithms.

source locations in the beamspace case are very close to each other and are located between the somatosensory and primary motor cortices, consistent with physiological expectations. While the mean source location is different for each algorithm and MT/ST-epochs combination, in general, the mean source locations tend to shift anterior for MUSIC and MVB and posterior for MLDF in the cases with greater variability.

We also evaluated the variability for source location estimates constrained to the cortical surface, that is, prior to performing the bounded unconstrained numerical optimization step. The constrained estimates show greater variability about the mean, and the source locations are not as physically consistent with somatosensory anatomy as the unconstrained estimates.

\section{DISCUSSION}

\section{A. Beamspace Transformation Design}

The transformation design procedure described in Section II-A provides large reductions in dimension for anatomically meaningful regions. The primary differences between the cases depicted in Fig. 4 lies in the response external to the ROI. As $M$ increases, the gain of the beamspace transformation external to the region increases. That is, reduced MSRE in the region is obtained at the expense of reduced spatial selectivity. The beamspace transformation for both 74- and 248-channel configurations passes signals from a fairly large region of the brain surrounding the somatosensory region. This suggests that the results are not likely to be sensitive to modest errors in registering the cortical surface or in choosing the ROI. That is, sources outside, but not too distant from the ROI are likely to be detected. This property is a negative attribute if sources outside the ROI are considered interference.

The ROI-based beamspace approach proposed here can be employed in the absence of prior information regarding the region of the brain of interest by using a variety of strategies. One is to tile the entire cortex with ROIs and perform beamspace processing in parallel on each. Another is a coarse-to-fine strategy where the ROI is successively refined based on the results of initial processing using a very large ROI.

The ratio of the number of statistically independent data samples to sample covariance matrix dimension is the primary factor affecting source localization variance. Note that sampling in time does not usually generate an equal number of statistically independent samples, especially if the data is filtered, since filtering causes successive samples to be correlated. As a rule of thumb, the ratio of independent data samples to covariance matrix dimension should exceed at least three to obtain reasonable performance (e.g., [4], [11], and [12]) with MVB. Consequently, beamspace transformations that significantly reduce the dimension potentially lead to dramatic performance improvements for limited data scenarios. For example, using the $M=13$ beamspace for the somatosensory region with the 248 channel Magnes 3600 Whole Head system results in a potential 19-fold reduction in data requirements under this rule of thumb. Even with limited prior information and choosing the ROI to be the entire hemisphere, a factor of 6 reduction (from 248 to 58) is obtained. Note that use of the local moment orientation does not significantly reduce the dimension of the beamspace transformation for anatomically significant ROIs. This is because the cortical surface is sufficiently convoluted that the orientation varies dramatically over the ROI, and consequently the moment effectively sweeps out the entire space spanned by the lead field matrices.

The spatial selectivity of the beamspace transformation is traded against source representation fidelity in the ROI by adjusting $M$. Using smaller values of $M$ leads to increased representation error within the ROI, which can result performance degradation relative to sensor space algorithms in the asymptotic case, when the covariance matrix is known. 
On the other hand, decreasing $M$ increases the attenuation experienced by activity that is outside the ROI, which can be beneficial for a number of reasons. For example, the MLDF algorithm used here assumes there is only a single source in the data. If the beamspace transformation sufficiently attenuates sources external to the ROI, then the single source assumption is relaxed to there only being one source in the ROI. Similarly, MVB is known to fail with correlated sources. If a correlated source located external to the ROI is sufficiently attenuated by the beamspace transformation, then beamspace MVB will correctly localize within the ROI [20]. However, design of highly selective transformations generally requires more precise prior information regarding expected source locations.

There are significant advantages to choosing the beamspace transformation in an optimal manner relative to ad hoc dimension reduction schemes such as throwing away sensor channels. Ad hoc methods generally result in asymptotic (known covariance matrix) performance loss. The transformation design procedure presented here minimizes the MSRE over a specified ROI for a given beamspace dimension. Other optimality criteria could be profitably employed for beamspace transformation design, especially if additional information, such as known locations of sources external to the ROI, is available.

\section{B. Beamspace Source Localization Performance}

The primary purpose of this study is to show that beamspace processing provides significant reductions in estimated source location variance. This conclusion is clearly demonstrated using the bootstrap method for all cases. The results also illustrate differences between the three different algorithms and two data selection procedures.

Note that SNR can be traded against the quantity of data for a given level of mean square localization error. That is, as SNR increases, the number of independent data samples required for a given mean square localization error decreases. Also, if the SNR and available data drop sufficiently low, the localization cost function is dominated by noise and estimated source locations are distributed throughout the brain in a random fashion. Threshold phenomena are common with modest SNR, that is, a small decrease in SNR may result in a large increase in mean square error [31]. The beamspace SNR is generally greater than the sensor space SNR because the beamspace transformation has negligible representation error for the signal and attenuates the noise that lies outside the space spanned by the columns of the transformation. Beamspace processing effectively increases the amount of data by reducing its dimension. Each algorithm has a different performance versus SNR and data sample tradeoff, a fact which is supported by our results.

Now consider the comparison between ST and MT cases. It appears at first glance that the MT case has significantly more data with which to estimate the noise subspace and covariance matrix. However, the MT case has only a slightly greater effective number of independent data samples than the ST case because the data is filtered. Note that a white low pass random process with $30-\mathrm{Hz}$ bandwidth requires more than $33.3 \mathrm{~ms}$ or 17 samples to reach the first null in its correlation function. Hence, the 21 samples used in the MT case for MUSIC and MVB are very strongly correlated and, thus, constitute approximately 1.2 statistically independent observations per epoch. MLDF employs data with $100-\mathrm{Hz}$ bandwidth for covariance matrix estimation and, thus, the MT case has approximately 2.1 statistically independent observations per epoch. The covariance matrix estimate for the MT case is, thus, at least as stable as the ST case since it has more data.

However, assuming the noise is stationary over the MT interval and the signal amplitude is approximated by the average depicted in Fig. 6, we conclude that the MT case has a lower effective SNR than ST because it estimates the sample covariance matrix by averaging over data on each side of the peak value of the evoked signal. If the signal component in the MT interval is approximated as a half cycle of a sinusoid, then the average signal power is $3 \mathrm{~dB}$ less than the peak power. This suggests that the difference in SNR between ST and MT could be several dB. Thus, performance differences are a consequence of MT having more data, but lower SNR.

Beamspace processing provides dramatic performance improvement with MLDF in both the ST and MT cases as shown in Fig. 8. The MLDF cost function for the ST sensor space case is well below threshold and results in randomly distributed source location estimates on the cortex, as illustrated in Fig. 7(a). In contrast, the beamspace ST estimates are very tightly clustered, even in the 50 epoch case. Note that the MT estimates are less variable than the ST estimates. This suggests that doubling the amount of data more than offsets the SNR loss, at least for the signal and noise levels in this data set.

MUSIC relies on separation between the estimated signal plus noise and noise only subspaces. With sufficient SNR, the subspaces converge much faster than the sample covariance matrix. Hence, sensor space MUSIC demonstrates much less variability than sensor space MLDF (or MVB) in the ST case, and beamspace MUSIC offers significant improvement relative to sensor space MUSIC only in the low data, 50 epoch scenario. However, with the lower SNR of the MT case, the subspaces are not estimated as well and higher variability results. In this case beamspace MUSIC has significantly less variability than sensor space MUSIC even with larger numbers of epochs. These results suggest that the higher SNR of the ST case more than offsets the reduction of data for MUSIC with this data set.

Fig. 10 shows that beamspace processing also results in significant reductions in estimator variability for MVB, especially when the number of epochs is less than 300. Note that MT is less variable than ST for sensor space processing, except for 300 epochs. In contrast, ST is less variable than MT in beamspace. These observations are explained as follows. In the 100 and 150 epoch sensor space cases the available data is small relative to the dimension, and increasing the amount of data by approximately $20 \%$ more than offsets the SNR loss of the MT case. However, in the 300 epoch sensor space case and in all the beamspace cases the amount of data is large relative to the dimension. Thus, increasing the amount of data by approximately $20 \%$ has much less impact on performance than increasing the SNR.

\section{SUMMARY}

Beamspace processing involves using a linear transformation to map the sensor space data into a lower dimensional space 
prior to applying a source localization (or other) algorithm. A procedure for designing a beamspace transformation that optimally represents a ROI is described and shown to be effective with common source localization algorithms. This design leads to significant reductions in dimension for meaningful anatomic structures.

Beamspace source localization offers the potential for significant reductions in estimated source location variability for algorithms that rely on estimated second order statistics or related quantities, especially in scenarios with limited data. The reduced variability of beamspace localization is illustrated for MLDF, MUSIC, and MVB by bootstrapping somatosensory data. While the exact benefit depends on the algorithm, SNR and amount of data, the performance improvement can be dramatic, especially when the SNR is low and a small number of independent data samples are available.

\section{REFERENCES}

[1] J. C. Mosher, P. S. Lewis, and R. M. Leahy, "Multiple dipole modeling and localization of spatio-temporal MEG data," IEEE Trans. Biomed. Eng., vol. 39, pp. 541-557, 1992.

[2] J. C. Mosher and R. M. Leahy, "Source localization using recursively applied and projected (RAP) MUSIC," IEEE Trans. Signal Process., vol. 47, no. 2, pp. 332-340, Feb. 1999.

[3] K. Sekihara, D. Poeppel, A. Marantz, H. Koizumi, and Y. Miyashita, "Noise covariance incorporated MEG-MUSIC algorithm: a method for multiple-dipole estimation tolerant of background brain activity," IEEE Trans. Biomed. Eng., vol. 44, no. 9, pp. 839-847, Sep. 1997.

[4] B. D. Van Veen, W. van Drongelen, M. Yuchtman, and A. Suzuki, "Localization of brain electrical activity via linearly constrained minimum variance spatial filtering," IEEE Trans. Biomed. Eng., vol. 44, no. 9, pp. 867-880, Sep. 1997.

[5] K. S. Sekihara, S. S. Nagarajan, D. Poeppel, A. Marantz, and Y. Miyashita, "Reconstructing spatio-temporal activities of neural sources using an MEG vector beamformer technique," IEEE Trans. Biomed. Eng., vol. 48, no. 7, pp. 760-771, Jul. 2001.

[6] S. E. Robinson and J. Vrba, "Functional neuroimaging by synthetic aperture magnetometry (SAM)," in Recent Advances in Biomagnetism, T. Yoshimoto, M. Kotani, S. Kuriki, H. Karibe, and N. Nakasato, Eds. Sendai, Japan: Tokio Univ. Press, 1999, pp. 302-305.

[7] R. D. Pascual-Marqui, "Standardized low resolution brain electromagnetic tomography (sLORETA): technical details," Meth. Findings Exp. Clin. Pharmacol., vol. 24, pp. 5-12, 2002.

[8] M. Scherg and D. von Cramon, "Two bilateral sources of the late AEP as indentified by a spatio-temporal dipole model," Electroencephalogr. Clin. Neurophysiol., vol. 62, pp. 32-44, 1985.

[9] S. Baillet, J. C. Mosher, and R. M. Leahy, "Electromagnetic brain mapping," IEEE Signal Process. Mag., vol. 18, no. 6, pp. 14-30, Nov. 2001.

[10] A. Dogandzic and A. Nehori, "Estimating evoked dipole responses in unknown spatially correlated noise with EEG/MEG arrays," IEEE Trans. Signal Process., vol. 48, no. 1, pp. 13-25, Jan. 2000.

[11] I. S. Reed, J. D. Mallett, and L. E. Brennan, "Rapid convergence rate in adaptive arrays," IEEE Trans. Aerosp. Electron. Syst., vol. AES-10, no. 6, pp. 853-863, Nov. 1974.

[12] K. A. Burgess and B. D. Van Veen, "Subspace based adaptive generalized likelihood ratio detection," IEEE Trans. Signal Process., vol. 44, no. 4, pp. 912-927, Apr. 1996.

[13] D. J. Chapman, "Partial adaptivity for the large array," IEEE Trans. Antennas Propag., vol. AP-24, no. 5, pp. 685-696, Sep. 1976.

[14] B. D. Van Veen and R. Roberts, "Partially adaptive beamformer design via output power minimization," IEEE Trans. Signal Process., vol. SP-35, no. 11, pp. 1524-1532, Nov. 1987.

[15] X. Guanghan, S. D. Silverstein, R. H. Roy, and T. Kailath, "Beamspace ESPRIT," IEEE Trans. Signal Process., vol. 42, no. 2, pp. 349-356, Feb. 1994.

[16] M. D. Zoltowski and T. S. Lee, "Maximum likelihood based sensor array signal processing in the beamspace domain for low angle radar tracking," IEEE Trans. Signal Process., vol. 39, no. 3, pp. 656-671, Mar. 1991.

[17] H. B. Lee and M. S. Wengrovitz, "Resolution threshold of beamspace MUSIC for two closely spaced emitters," IEEE Trans. Signal Process, vol. 38, no. 9, pp. 1545-1559, Sep. 1990.
[18] M. D. Zoltowski, G. M. Kautz, and S. D. Silverstein, "Beamspace rootMUSIC,” IEEE Trans. Signal Process, vol. 41, no. 1, pp. 344-364, Jan. 1993.

[19] W. F. Gabriel, "Using spectral estimation techniques in adaptive processing antenna systems," IEEE Trans. Antenna Propag., vol. AP-34, no. 3, pp. 291-300, Mar. 1986.

[20] B. D. Van Veen, A. Rodríguez-Rivera, and R. T. Wakai, "MEG source localization via partially adpative LCMV," in Proc. 13th Int. Conf. Biomagnetism, Aug. 2002, pp. 731-733.

[21] E. Efron and R. J. Tibshirani, An Introduction to Bootstrap. London, U.K.: Chapman \& Hall, 1993.

[22] F. Darvas, M. Rautiainen, S. Baillet, A. Ossaidtchi, J. C. Mosher, and R. M. Leahy, "Investigations of dipole localization accuracy in MEG using bootstrap," in Proc. 2003 IEEE Workshop Statistical Signal Processing, St. Louis, MO, Sep. 28-Oct. 1 2003, pp. 282-285.

[23] J. Gross and A. A. Ioannides, "Linear transformations of data in space in MEG," Phys. Med. Biol., vol. 44, pp. 2081-2097, 1999.

[24] S. Taulu, M. Kajola, and J. Simola, "Suppression of interface and artifacts by the signal space separation method," Brain Topogr., vol. 16, pp. 269-275, 2004.

[25] S. S. Dalal, J. M. Zumer, K. Sekihara, and S. S. Nagarajan, "Modified beamformers for coherent source region suppression," in Proc. 14th Int. Conf. Biomagnetism, 2004, p. 488.

[26] B. Fischl and A. Dale, "SURFER: a software package for cortical surface based analysis and visualization," NeuroImage, vol. 9, no. 1, p. S243, 1999.

[27] B. V. Baryshnikov, B. D. Van Veen, and R. T. Wakai, "Maximum likelihood estimation of low rank signals for multiepoch MEG/EEG analysis," IEEE Trans. Biomed. Eng., vol. 51, no. 11, pp. 1981-1993, Nov. 2004.

[28] E. J. Kelly and K. M. Forsythe, "Adaptive Detection and Parameter Stimation for Multidimensional Signal Models," Lincoln Laboratory, Cambridge, MA, Tech. Rep. 848, 1989.

[29] K. S. Sekihara, S. S. Nagarajan, D. Poeppel, and A. Marantz, "Asymptotic SNR of scalar and vector minimum-variance beamformers for neuromagnetic source reconstruction," IEEE Trans. Biomed. Eng., vol. 51, no. 10 , pp. 1726-1734, Oct. 2004.

[30] R. Hari and R. J. Ilmoniemi, "Cerebral magnetic fields," CRC Crit. Rev. Biomed. Eng., vol. 14, pp. 93-126, 1986.

[31] C. D. Richmond, B. D. Van Veen, and T. Limpiti, "Mean squared error performance prediction of linearly constrained minimum variance source localization," in Proc. 15th Int. Conf. Biomagnetism, Aug. 2004, pp. 581-582.

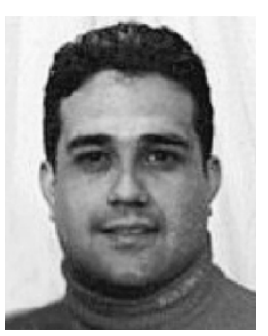

Alberto Rodríguez-Rivera (S'96-M'05) was born in Mayaguez, Puerto Rico, in 1969. In 1992, he received the B.S. degrees with honors (magna cum laude) both in electrical engineering and computer engineering from the University of Puerto Rico-Mayaguez (UPRM). In 1995, He received the M.S. degree from the UPRM and in 2004 the Ph.D. degree from the University of Wisconsin-Madison both in electrical engineering.

Since the Spring of 2005, he has been with the Department of Electronics and Electrical Engineering Techonology at the Madison Area Technical College. His research interests include multidimensional, array, and statistical signal processing, its biomedical applications and implementation.

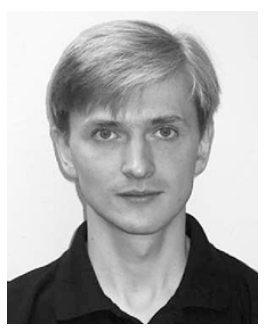

Boris V. Baryshnikov was born in Novgorod, Russia. He received the undergraduate degree with honors in physics/biophysics in 1997 from Moscow State University, Moscow, Russia. In 2000 and 2001, he received the M.S. degrees in medical physics and computer science, respectively, from the University of Wisconsin, Madison, WI. In 2004, he received the Ph.D. degree in medical physics while working under the supervision of Prof. R. T. Wakai in the biomagnetism laboratory at the University of Wisconsin-Madison.

Currently, he is working for Microsoft Corp. His research interests include biomedical signal processing with emphasis on practical applications and development of algorithms and tools for data analysis and visualization. 


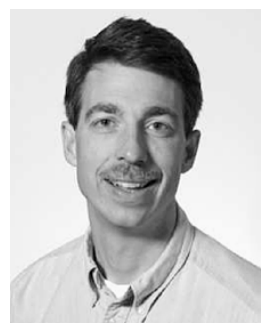

Barry D. Van Veen (S'81-M'86-SM'97-F'02) was born in Green Bay, WI. He received the B.S. degree from Michigan Technological University, Houghton, in 1983 and the Ph.D. degree from the University of Colorado, Boulder, in 1986, both in electrical engineering. He was an ONR Fellow while working on the Ph.D. degree.

In the spring of 1987, he was with the Department of Electrical and Computer Engineering at the University of Colorado, Boulder. Since August of 1987, he has been with the Department of Electrical and Computer Engineering at the University of Wisconsin-Madison and currently holds the rank of Professor. His research interests include signal processing for sensor arrays, magneto- and electroencephalography, and biomedical applications of signal processing.

Dr. Van Veen was a recipient of a 1989 Presidential Young Investigator Award from the National Science Foundation and a 1990 IEEE Signal Processing Society Paper Award. He served as an associate editor for the IEEE TRANSACTIONS ON Signal PROCESSING and on the IEEE Signal Processing Society's Statistical Signal and Array Processing Technical Committee and the Sensor Array and Multichannel Technical Committee. He received the Holdridge Teaching Excellence Award from the ECE Department at the University of Wisconsin in 1997. He coauthored "Signals and Systems," (1st Ed. 1999, 2nd Ed., 2003, New York: Wiley) with S. Haykin.

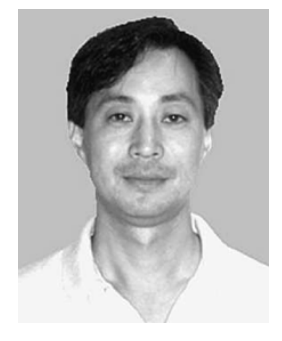

Ronald T. Wakai was born in East Orange, NJ, in 1958. He received the B.A. degree with honors in physics from Cornell University, Ithaca, New York in 1980 and the Ph.D. degree in physics from the University of Illinois, Urbana, Illinois in 1987.

Since then, he has been with the Department of Medical Physics at the University of Wisconsin, Madison, Wisconsin, where he is currently a Professor. His research interests include basic and technical aspects of fetal biomagnetism and adult MEG. 